\title{
INFLUENCE OF DRUGS AFFECTING THE AUTONOMIC SYSTEM ON SEMINAL EJACULATION
}

\author{
PHILIP J. DZIUK AND H. W. NORTON \\ 111 Animal Genetics, Department of Animal Science, \\ University of Illinois, Urbana, Illinois, U.S.A.
}

(Received 25th October 1961)

\begin{abstract}
Summary. Rabbits and boars were used to study the effect on the ejaculation process of various drugs known to affect the autonomic nervous system. Of those tried, only atropine had an effect, causing linear reduction, as the dose increased, of the logarithm of the number of spermatozoa ejaculated by rabbits. In the boar, the volume of semen was reduced, but at the dose levels employed total spermatozoa per ejaculate changed little. This suggests that the male accessory sex glands are partly controlled by the parasympathetic system and that this system also has an influence on spermatozoon movement from the epididymis through the vas deferens.
\end{abstract}

\section{INTRODUCTION}

A normal single seminal ejaculate usually contains many more spermatozoa than are found in the vas deferens, but fewer than in the epididymis (Chang, 1946; Chang \& Sheaffer, 1957; Baily \& Smith, 1958; unpublished observations of the authors). The majority of spermatozoa appearing in ejaculates must then be transported the entire length of the vas deferens in a relatively short period of time just prior to or at ejaculation. Several investigations (Cross \& Glover, 1958; Macmillan \& Auckland, 1960) have led to an understanding of spermatozoon transport in the epididymis, but relatively little information is available on the mechanism of spermatozoon transport at ejaculation. A number of authors (Bacq, 1931; Gunn, 1936; Cross, 1959, and others) have divided the ejaculation process into two phases, erection and ejaculation. The parasympathetic system is given a dominant role in erection, while ejaculation and accessory gland secretion are attributed to the sympathetic system. This report is of an attempt to assess the role of the autonomic system in moving spermatozoa within the male.

\section{MATERIALS AND METHODS}

Sixteen male Dutch-type rabbits ( 4 to $8 \mathrm{lb}$ ), 1 to 3 years old, trained to serve an artificial vagina, were the experimental animals. Each male was housed separately in either inside or outside hutches. Each male served as his own control, by being subjected to regular semen collections between treatments. 
This was done in an attempt to minimize errors of interpretation due to dayto-day variations within rabbits (Doggett, 1956). Semen collections were made every 7 to 10 days and at each collection an attempt was made to obtain a series of four ejaculates. The first ejaculate was obtained about 20 min after injection of the drug to be tested to allow time for absorption and effect. Subsequent ejaculates were then collected at approximately 15-min intervals.

Spermatozoa in the second, third and fourth ejaculates would then come from the epididymis during the time the drug was acting, so any effect of the drug on spermatozoon transport should be reflected in the numbers of spermatozoa in these ejaculates. The drugs to be tested were ordinarily injected subcutaneously in 0.1 to $0.2 \mathrm{cc}$ of physiological saline solution. Drugs and dosages per male are as follows: atropine sulphate, $0 \cdot 125,0 \cdot 188,0 \cdot 972,1 \cdot 30$, $\mathrm{I} .62$ or $3.24 \mathrm{mg}$; carbachol, 0.04 or $0.075 \mathrm{mg}$; dibenamine, 20 or $40 \mathrm{mg}$; purified oxytocin preparation, 10 or 50 units. The total number of spermatozoa in each ejaculate was determined following the addition to the ejaculate and the washings from the artificial vagina of sufficient fluid to suspend the ejaculate in $10 \mathrm{ml}$. The fluid used for suspension was a $5 \%$ sodium citrate solution to which a small amount of formalin had been added. The hypertonic citrate solution effectively dispersed the non-spermatozoon semen components, facilitating visualization at counting in a haemocytometer chamber. At least 200 spermatozoa were counted in each sample.

The logarithm of the total number of spermatozoa per ejaculate was analysed statistically to isolate variations associated with individuals, treatments and dose levels.

Three boars weighing 285,300 and $350 \mathrm{lb}$, previously trained to mount a dummy and serve an artificial vagina, were used to determine the influence of atropine on ejaculation in a species producing a large volume of semen $(200$ to $500 \mathrm{ml})$, a large number of spermatozoa per ejaculate $\left(20\right.$ to $\left.80 \times 10^{9}\right)$ and having a prolonged ejaculation time (5 to $15 \mathrm{~min}$ ). Atropine sulphate was dissolved in $4 \mathrm{ml}$ of saline solution and injected subcutaneously behind one ear. The levels of atropine tested were $32.4,16 \cdot 2$ or $8 \cdot 1 \mathrm{mg}$ per boar. The injection was made without restraining the boar while his attention was diverted to a small amount of feed. Semen collection commenced about $30 \mathrm{~min}$ after injection to permit absorption and effect. Semen collections were made at 4-day intervals and atropine injections were made every 12 days so that each boar served as his own control. Spermatozoa were counted by essentially the same procedure as outlined for rabbits.

\section{RESULTS}

\section{RABBITS}

General and copulatory behaviour in rabbits was affected very little by treatment, with the exception of the oxytocin preparation. About 30 to $60 \mathrm{~min}$ after the injection of 50 units of oxytocin, the rabbits often became prostrate and unresponsive, with rapid shallow breathing. It is likely that components of the posterior pituitary extract, other than oxytocin, particularly vasopressin, caused this response. In some cases, rabbits receiving a high dose of atropine had 
difficulty in penetrating the artificial vagina due to an insufficient erection. Rabbits on all but the lowest doses of atropine had impaired ability to constrict the pupil in response to a bright light. Vision did not seem to be affected.

Nine hundred ejaculates were collected from the rabbits. Statistical analysis revealed no effect by any drug except atropine. As the dose increased, atropine caused a linear decrease in the logarithm of the number of spermatozoa ejaculated.

\section{BOARS}

Copulatory behaviour in treated boars as evidenced by their reactions towards the dummy sow, thrusting, erection and duration of ejaculation was essentially normal. In some cases, erection was not maintained at the usual level throughout the ejaculation. Vision in bright outdoor light was apparently unimpaired by the atropine doses used. Salivation and foaming at the mouth, which often accompany mating in the boar, were either absent or less than usual. No persistent after-effect of any kind was noted even after repeated treatments.

The volume of liquid semen produced by atropine-treated boars was about

TABLE 1

SEMEN GHARAGTERISTICS IN THE BOAR FOLLOWING ADMINISTRATION OF ATROPINE

\begin{tabular}{c|c|c|c|c}
\hline \multicolumn{3}{c|}{ Vol. liquid semen per ejaculate $(c c)$} & \multicolumn{2}{c}{ No. sperm per ejaculate $\left(\times 10^{9}\right)$} \\
\hline Boar & Control & Treated & Control & Treated \\
\hline D & $148 \pm 47$ & $42 \pm 23$ & $47 \pm 18$ & $57 \pm 29$ \\
H & $200 \pm 41$ & $27 \pm 21$ & $25 \pm 7$ & $20 \pm 14$ \\
HN & $135 \pm 50$ & $37 \pm 29$ & $37 \pm 12$ & $43 \pm 28$ \\
\hline
\end{tabular}

$\frac{1}{4}$ of normal (Table 1). The total number of spermatozoa per ejaculate was the same as normal. As a result, the semen was very white and opaque due to the high concentration of spermatozoa. Very little of the tapioca-like material or other accessory-gland fluids was present. At any given dose the response seemed to be inversely related to the weight of the boar.

\section{DISCUSSION}

As no gross side effect of atropine was noticed, it is concluded that inhibiting the parasympathetic portion of the male reproductive system reduces the number of spermatozoa available at each ejaculation in the rabbit, the logarithm changing linearly with the dose.

The smaller-than-normal volume of semen from boars following treatment with atropine indicates inhibition of accessory-gland secretion. The doses of atropine employed apparently inhibited accessory-gland secretion without significantly interfering with spermatozoon transport. Based on the observation at autopsy that the seminal vesicles of the boar have a considerable amount of preformed secretions, and that the prostate and bulbourethral glands have little 
secretion present, it seems that atropine prevents excretion of preformed materials as well as secretion. Thus the parasympathetic system exerts an influence first on the male accessory sex organs and secondarily on the movement of spermatozoa through the vas deferens. This does not preclude the possibility that the sympathetic system exerts the influence usually ascribed to it.

McKenzie, Miller \& Bauguess (1938) determined the concentration of spermatozoa in the epididymis of the boar to range from 4.6 to $6.8 \times 10^{9}$ per $\mathrm{ml}$. On this basis most of the semen samples obtained after atropine treatment contained one part of epididymal contents to two to five parts of accessorygland secretion, in contrast to normal boar semen in which epididymal contents usually constitute less than one part in fifty.

Semen samples with less than the normal amount of accessory-gland secretions and having a higher-than-normal concentration of spermatozoa may be useful in semen studies and for lessening problems associated with storing the usual large volume of boar semen.

\section{ACKNOWLEDGMENTS}

This work was supported in part by a grant from The National Academy of Science, National Research Council Committee on Sex.

The assistance of L. James, L. J. Hirsch, J. Gladish and B. Dodds is gratefully acknowledged.

\section{REFERENCES}

BAcQ, J. M. (1931) Impotence of the male rodent after sympathetic denervation of the genital organs. Amer. F. Physiol. 96, 321.

BaILY, G. \& Smith, V. R. (1958) Number of spermatozoa in the different parts of the reproductive tract of the bull. Dairy Sci. 41, 1781.

Chang, M. C. (1946) The sperm production of adult rams in relation to frequency of semen collection. 7. agric. Sci. 35, 243.

Chang, M. C. \& Sheaffer, D. (1957) Number of spermatozoa ejaculated at copulation, transported into the female tract and present in the male tract of the golden hamster. 7. Hered. 48, 107.

CRoss, B. A. (1959) Hypothalamic influences on sperm transport in the male and female genital tract. Endocrinology of Reproduction, p. 167. Ed. C. W. Lloyd, Academic Press, New York.

Cross, B. A. \& Glover, T. D. (1958) The hypothalamus and seminal emission. F. Endocrin. 16, 385.

Doggett, V. C. (1956) Periodicity in the fecundity of male rabbits. Amer. F. Physiol. 187, 445.

GunN, M. C. (1936) Artificial production of seminal ejaculation and the characters of spermatozoa contained therein. Bull. Council sci. $E^{\circ}$ indust. Res. No. 94.

Macmillan, E. W. \& Auckland, J. (1960) The transport of radiopaque medium through the initial segment of the rat epididymis. F. Reprod. Fertil. 1, 139.

MaKenzie, F. F., Miller, J. C. \& Bauguess, L. C. (1938) The reproductive organs and semen of the boar. Mo. Agric. exp. Sta. res. Bull. p. 279. 